\title{
Vuelta al origen. Una propuesta de aproximación al archivo desde la teoría matricial
}

\author{
Garazi Ansa Arbelaiz \\ Universidad del País Vasco \\ garaziansa@gmail.com
}

RESUMEN: Durante los últimos decenios han sido varias las tentativas de cuestionar los grandes relatos hegemónicos y patriarcales desde el arte a partir de posicionamientos feministas. Dentro de estos intentos, caben destacar los realizados desde la práctica comisarial, específicamente en los que se ha utilizado el archivo como método expositivo. Mediante este estudio, analizamos esta última práctica a través del caso de estudio Anarchivo Sida, donde el archivo aparece como medio potencial que posibilita agrietar esos discursos oficiales y unidireccionales, a la vez que cuestionamos el uso o la aplicación de las grandes teorías patrilineales sobre el concepto del archivo utilizados para ello. Así, presentamos la importancia del uso de unas teorías adecuadas como base de los proyectos comisariales y sus instalaciones, y proponemos una nueva aproximación al archivo aplicable al ámbito expositivo desde la teoría matricial de Bracha L. Ettinger que se articula desde lo femenino.

PALABRAS CLAVE: Archivo; Instalación; Feminismos; Discursos históricos; Bracha L. Ettinger.

\section{Back to the Source. An Approximation Proposal to the Archive from the Matrix Theory}

ABSTRACT: During the last decades there have been several attempts to question the hegemonic and patriarchal stories from art based on feminist stances. Among these attempts, those made from the curatorial practice, specifically those in which the archive has been used as an exhibition method, are worth highlighting. In this study, we analyze this last practice through the case study Anarchivo Sida, where the archive works as a potential tool that makes it possible to crack those official and unidirectional discourses, while questioning the use or application of the patrilineal theories about the concept of the archive used for it. Thus, we present the importance of the use of adequate theories as a basis for the curatorial projects and their installation, and we propose a new approach to the archive, relevant to the exhibition area, from the Bracha L. Ettinger's matrix theory that is articulated from the feminine.

KEYWORDS: Archive; Installation; Feminisms; Historical discourses; Bracha L. Ettinger.

Recibido: 28 de febrero de 2018 / Aceptado: 1 de febrero de 2019.

El visitante entraba a la sala de exposiciones y se encontraba abrumado por la avalancha de información que recaía ante sus pupilas. El espacio estaba inundado de documentos, de muebles, y de proyectos artísticos que se amalgamaban. Esta sería una breve aproximación descriptiva de una de las salas expositivas de Tabakalera-Centro Internacional de Cultura Contemporánea ${ }^{1}$ de Donostia-San Sebastián en la primavera del año 2016, donde se instaló Anarchivo Sida, una tentativa expositiva del resultado de una investigación llevada a cabo por el Equipo $\mathrm{Re}^{2}$.

Dicho proyecto tenía como objetivo principal analizar, identificar y recopilar «la producción cultural, visual y performativa, entorno al VIH/sida, atendiendo, por vez primera, a las prácticas desarrolladas fuera del marco hegemónico» (Arriola, Garín y Valdés, 2016: 21), centrándose en los casos de Chile y de España. Así, el objetivo de dicho proyecto era acercarse al cuerpo enfermo como condicionante de activación hacia un nuevo cambio del paradigma, sobre todo visual, pero también afectivo y económico (Arriola, Garín y Valdés, 2016).

Cómo citar este artículo: GARAZI ARBELAIZ, Ansa, "Vuelta al origen. Una propuesta de aproximación al archivo desde la teoría matricial», Boletín de Arte-UMA, n. ${ }^{40,}$ Departamento de Historia del Arte, Universidad de Málaga, 2019, pp. 255-265, ISSN: 0211-8483, e-ISSN: 2695-415X, DOI: http://dx.doi.org/10.24310/BoLArte.2019. v0i40.5492 
El resultado de esta investigación, constituido por un compendio de documentos de muy diferente naturaleza, fue presentado en la exposición con la forma de un archivo. En este artículo analizaremos las definiciones y teorías sobre las que el Equipo Re se basó a la hora de corporalizar los documentos en este archivo, estudiaremos su adecuación al proyecto -incluyendo su instalación-, y propondremos una nueva y posible aproximación a su lectura, partiendo de la teoría matricial.

Pero antes de comenzar en la profundización de las singularidades del proyecto mencionado, es menester abordar otros temas pertinentes a la cuestión, que nos posibilitarán un acercamiento más preciso al análisis posterior. Analizaremos diferentes aproximaciones teóricas al concepto del archivo, las posibilidades que genera, y plantearemos la importancia del acercamiento a dicho concepto desde nuevas posiciones teóricas atravesadas por las teorías feministas, y las posibilidades que podría generar al aplicarlas en contextos expositivos.

Históricamente, se han proyectado unas características específicas y un orden social determinado sobre el pasado creando un canon que a su vez ha regido el presente y el futuro. Se trataba de un canon que, a través de diferentes mecanismos de exclusión, ha logrado imponer la figura del hombre blanco occidental como centro de la sociedad, y ha creado así una hegemonía social de clase, raza y género, configurando al Otro en clara contraposición a la norma dominante (Pollock, 1999: 3-21). Uno de los mayores movimientos de lucha emancipadora frente a la imposición de dicho orden social, ha sido propulsado por los movimientos feministas - gracias sobre todo a la segunda y tercera ola-, cuyo objetivo principal ha sido asumir, según lo menciona Mulvey, «un proyecto político transformador, basado en una interpretación ampliada de la injusticia y una crítica sistémica a la sociedad capitalista» (Mulvey, 2001: 252), seguida por el cuestionamiento de la construcción y establecimiento de las estructuras que propiciaban la pervivencia de dicho orden.

La disciplina de la historia del arte no ha sido una excepción en cuanto a estos hechos, pues el canon hegemónico ha infectado toda su estructura y construcción de la misma forma, incluso arraigándose en ella en más profundi$\mathrm{dad}^{3}$. A pesar de ello, y de nuevo gracias a los movimientos y teorías feministas, sobre todo de los últimos 40 años, ha habido grandes tentativas -que además van en aumento- de cuestionar y visibilizar la gran ficción que ocultan todos estos discursos naturalizados y que han construido la historia del arte hegemónica y androcéntrica; estas se han llevado a cabo desde las áreas más teóricas ${ }^{4}$ pero también desde la práctica artística y expositiva.

Cabe resaltar que en los últimos años, sobre todo desde la práctica comisarial y artística y especialmente en relación a las teorías feministas, se ha producido un acercamiento a la metodología del archivo como formalización expositiva $^{5}$. Una posible razón para esta aproximación podría ser la oportunidad de translucidez que proporciona (Ansa, 2017). Resulta paradójico, sin embargo, que dicha aproximación se centre en torno a un concepto de archivo fundamentado sobre discursos postestructuralista y no desde los feminismos, donde los nombres de Derrida y Foucault nunca faltan.

En los apartados siguientes nos centraremos en el análisis de la interrelación entre el archivo y el canon con la intención de vislumbrar las posibles contradicciones que puede suscitar utilizar dichas teorías en consonancia con las teorías feministas, y propondremos una nueva y posible aproximación al concepto archivístico desde lo matricial que será aplicable a los espacios expositivos.

\section{Desde la mirada hacia el cuerpo}

\section{Espacios de ley y norma}

El archivo se ha asociado de forma tradicional a la escritura patriarcal y androcéntrica de la historia, y por la misma razón se puede también asociar a la construcción y hegemonía del canon, como veremos más adelante. Esta asociación se convirtió en una relación indisociable en especial a partir del siglo XIX, pues el archivo se constituyó como el repositorio de hechos tanto materiales, como simbólicos, sobre los cuales se asentaba la escritura y el imaginario histórico social, nacional y territorial (Spivak, 2006: 164 y Morandeira; 2016: 3-4). Así, desde entonces, y junto a los museos, su objetivo principal ha sido articular el orden, las leyes y normas a seguir de la sociedad europea occidental (Bennet, 1998), a través de crear y difundir la idea de que el conocimiento y orden dados eran los universales, naturales y científicos. Aunque en realidad se trataba de la imposición de la 
mirada de la clase burguesa masculina occidental, de una ficción disfrazada de objetividad que había sido construida mediante fragmentos específicos seleccionados de los archivos (Spivak, 2006).

En el caso de este último, es relevante subrayar que su gran eficiencia en dicha labor se ha articulado a través de la violencia ${ }^{6}$ (Morandeira, 2016: 3), es decir, a través de una forma muy específica de interacción -entre los sujetos y hechos-, que está basada a su vez en la ejecución directa del poder jerárquico; la misma interacción sobre la que se sustenta de igual manera y desde sus orígenes mismos el patriarcado (Lerner, 2018). Así, se puede afirmar que es gracias a esta violencia ejercida, por lo que el concepto y función del archivo, así como del patriarcado, han sido invariables y se han mantenido firmes durante tantas décadas, protectores de un pasado inmutable e impenetrable. Es más, esta afirmación va acorde a la propuesta que presenta Derrida en su ya clásico ensayo Mal de archivo, en el que subraya el carácter autoritario del archivo, y su vinculación al poder y a la norma, al definirlo como lugar de origen (1997).

Y precisamente es en el origen de su significado donde se vinculan los conceptos de archivo y canon, pues tal y como expone Pollock, al igual que el primero, el canon también estaba asociado a la idea de «norma» y «ley» (1999: 3). El canon es el eje central que legitima retrospectivamente la identidad política y cultural, una narración consolidada, que confiere autoridad y naturaliza su función; es lo incuestionable (Pollock, 1999: 3). De esta forma no cabe duda, que tanto el archivo como el canon son dos instituciones relacionadas desde el mismo origen con el poder de la escritura -androcéntrica y hegemónica-, la norma y la autoridad. Y por esta misma razón, llama la atención la escasa cantidad de debates y cuestionamientos que ha habido sobre el concepto del archivo desde las nuevas teorías feministas sin haber habido apenas propuestas de nuevos enfoques para articular nuevos discursos sobre su potencialidad, en comparación al concepto del canon?

Ha habido algún un intento de desenmascaramiento y crítica bastante laxo, entre los cuales se podría destacar la propuesta de Pollock con el término "patriarchivo»8 (2008: 44), o las recientes aportaciones de Suely Rolnik, las cuales esclarecen el porqué del auge del uso del archivo en las prácticas artísticas y expositivas actuales, vinculando su creación a una sensación de un malestar represivo transge- neracional (bien en forma de la violencia Estatal, la patriarcal o la represión colonial) que ha hecho surgir la necesidad de hacer visible las otras narrativas que dentro de las grandes historias-hegemónicas no tenían lugar, y que por lo tanto habían sido desaparecidas. De esta forma el interés de la aportación de Rolnik radica en que estas prácticas de archivo son entendidas por primera vez no como prácticas de poder, sino como prácticas de sanación frente a los traumas y diferentes tipos de violencias y malestares sufridas en el pasado ${ }^{9}$.

A pesar de la interesantísima aportación de Rolnik, el autor que más frecuentemente se sigue mencionando desde los feminismo a la hora de aproximarse al concepto archivístico es Foucault, que lo presenta como un sistema activo de una estructura totalmente descentrada capaz de regular la creación y aparición de múltiples e infinitos enunciados; un sistema inabarcable en su totalidad, lo que concluye que cada significado y enunciado creado a partir del mismo tendrá un carácter totalmente fragmentario, parcial y equitativo (Foucault, 2002: 219-222). La ventaja de esta definición de la que hacen uso los feminismos es que presenta el archivo como una nueva estructura para la reconstrucción del pasado y el cuestionamiento de la hegemonía y canon históricos, ofreciendo la oportunidad de crear diferentes historias paralelas, complementarias o discordantes, todas válidas y equitativas, ya que preservan el principio de la credibilidad, que le concede autoridad y el derecho a ser escuchado ${ }^{10}$. A pesar de ello, el gran problema de la aportación de Foucault es que en ningún momento define la forma de relacionarse de los usuarios del archivo con el archivo mismo, por lo que en ningún momento cuestiona el paradigma universal del acceso al conocimiento que se sustenta en el sistema cognitivo y perceptivo, es decir, en la mirada, sino que de alguna manera la perpetúa.

Una puesta en escena: Anarchivo Sida

Una de las propuestas planteadas durante estos últimos años y que se vincula a la perfección con los conceptos anteriormente mencionados es el proyecto Anarchivo Sida. Se trata de un proceso de investigación de larga duración llevada a cabo por el colectivo Equipo Re, que dio comienzo entre los años 2012 y 2013 en el marco de las Residencias 
de Investigación del Museo Reina Sofía en Madrid, y que actualmente se sigue desarrollando. El punto de partida de dicha investigación es la llamada "crisis del sida» que se origina hacia finales de los años 80 , que se propone como punto de partida para explorar las diferentes estrategias críticas y modelos que se activaron a su alrededor. Específicamente se centran en el análisis de las prácticas estéticas, modelos performativos y representaciones con gran centralidad en el cuerpo que pueden rastrearse hasta la actualidad. Así su intención es acorde a las teorías feministas: trazar los nuevos desplazamientos, cambios y fragmentaciones que aparecieron en el ámbito y la estructura misma de la producción cultural y el arte, centrándose además, según una geolocalización no hegemónica, en Chile y España ${ }^{11}$.

Este proceso de investigación ha tenido dos fases. Durante la primera, entre los años 2012-2014, se recopilaron y se produjeron diferentes materiales a través de diversas acciones de investigación y debate, con las que conformaron un archivo. La segunda fase, y la que más nos interesa en este estudio, realizada entre 2015-2017, se correspondió a la activación y visibilización del proyecto por doble vía: por medio de una plataforma web ${ }^{12}$, donde se exponen varios y diferentes documentos relacionados con el proyecto; y por otra parte, a través del formato expositivo en forma de archivo. A pesar de que el Anarchivo Sida fue expuesto por primera vez en la muestra colectiva Based on a True Story - A.I.D.S., realizada en The Deutsche Hygiene Museum, en Dresden (2015), el objeto de estudio de este análisis será la exposición individual del proyecto realizada en Tabakalera de Donostia-San Sebastián en la primavera del 2016.

La exposición fue articulada en torno a tres ejes temáticos vinculados al contexto de la "crisis del sida» a partir del binomio naturaleza/cultura: la división histórica entre el ser humano y el animal; la muerte desde una perspectiva cultural; y la salud como convención jerárquica que divide los cuerpos entre normales y enfermos. Tres ejes temáticos cuyo núcleo está directamente relacionado con el cuerpo humano, su gestión política y su representación histórica y pública (Arriola, Garín, y Valdés, 2016: 22), que a su vez fueron cruciales para su posterior instalación. El diseño se realizó a través de la colaboración con la artista Carme Nogueira, para quien la visibilidad de los dispositivos es un tema de especial interés, ya que según la artista, a pesar de que son elementos que crean significados, nuestras miradas están tan acostumbradas a ellos que automáticamente se vuelven transparentes ante nuestros ojos. Por esta razón, Nogueira busca subrayar su presencia, para que el visitante sea consciente de ellos y los interprete y experimente junto al archivo expuesto ${ }^{13}$.

La intención de la artista fue crear diferentes soportes para las piezas-documentos que componían el archivo según los tres ámbitos temáticos en cuales se estructuraba la exposición, -lo animal, la muerte y la salud-, ya que cada uno de ellos le sugería una posición física diferente del cuerpo-espectador. Para ello, realizó los dispositivos-muebles a diferentes alturas que obligaban a los cuerpos a agacharse -para acercarse al nivel animal-, a distanciarse -relacionado a la muerte- o a imponerse -vinculado a la salud-. De esta forma, su intención era que el modo de acercamiento de los visitantes hacia los dispositivos se convirtiera en una acción totalmente consciente ${ }^{14}$.

Todos estos dispositivos fueron realizados con madera OSB y usando la medida estándar del tablón casi sin manipularlo, jugando con sus posiciones: en horizontal como mesa baja, vertical para simular un tablón de anuncios e inclinada como mesa-expositor.

Como resultado, el diseño expositivo, tal y como Nogueira esperaba, obligaba a los visitantes a adoptar diferentes posiciones y posturas a la hora de acercarse a los documentos. La intención de visibilizar el dispositivo había funcionado, pero tal vez había funcionado demasiado bien. Una vez dentro de la sala, el visitante, más que tener la sensación de estar andando dentro de un archivo, tenía la de estar experimentando una instalación. Así, el proyecto de Nogueira, en vez de adquirir el formato de un dispositivo, adquirió la identidad de una obra artística, relegando el archivo a un segundo plano. Pero el cambio del estatus del dispositivo no solamente transformó el comportamiento del visitante respecto a los soportes, sino que también hacia los documentos expuestos, ya que al adquirir el estatus de obra de arte los visitantes se acercaban a los dispositivos, aunque no demasiado por miedo a tocarlos, y los observaban mientras los cuerpos simplemente paseaban entre los pasillos creados entre los muebles. Por inercia, los ojos de los visitantes se posaban sobre los diferentes flyers, fotos y fotocopias que había sobre los soportes, pero su protagonismo parecía secundario bajo la monumentalidad de los paneles de madera. Así, activar los documentos y el archivo por par- 
te de los visitantes -acción imperativa para crear enunciados (Ansa, 2017)-, se convertía en una tarea ardua, puesto que la mirada se encontraba sobreestimulada, y a los documentos solo se podía acceder a través de los ojos, y no a través del cuerpo.

Llegados a este punto, es interesante recuperar los teóricos en los que el Equipo Re se había basado para conformar el formato archivístico para la exposición, para así poder profundizar en las razones para la elección de una instalación donde toda la responsabilidad recaía solo en la mirada. Sus grandes referentes fueron Derrida y Foucault ${ }^{15}$. A estos dos nombres hay que añadir el de Pedro G. Romero, ya que incluso el título mismo del proceso y de la exposición fue apropiada de su posicionamiento sobre el archivo, que propone una antítesis del mismo: el anarchivo. Así, el anarchivo indicaría la «ausencia de apriorismo», la «ausencia de jerarquías», un no-archivo que fuera en contra del archivo oficial que, siguiendo las ideas foucaultianas, es organizado mediante huecos y sinuosidades, repeticiones y divergencias (Dressler, 2016).

A pesar de que nos pueda asombrar que entre sus referentes no se encontraba la figura de Rolnik ya que sin ninguna duda la "crisis del sida» fue una época traumática y violenta, desde un punto de vista teórico, en general, parece que los teóricos mencionados aportan claves para llevar a cabo la intención del proyecto Anarchivo Sida de trazar nuevas historias, desplazamientos, de cuestionar las estructuras de la producción cultural y del arte y las grandes hegemonías. Para ello, la estrategia que se aplica es la centralidad del cuerpo, articulando las políticas del cuerpo con las políticas del archivo. ¿Pero en todo esto, dónde queda el cuerpo?

Su importancia ha quedado patente en cuanto al diseño expositivo, pero en cuanto a la noción del archivo queda totalmente disperso. Ni las teorías de Derrida, ni las de Pedro G. Romero, ni las de Foucault toman en consideración la importancia del cuerpo en relación al archivo. Todas ellas analizan y determinan el archivo como un ente aislado, apenas en contacto con el cuerpo humano, si cabe, solo con su mirada. Al analizar las teorías de Foucault queda patente la importancia que le da el autor a la asociación e interconexión de los diferentes documentos inscritos dentro del archivo. Sin embargo, tal y como hemos expuesto antes, no analiza ni estudia la propia acción, es decir, cómo se llevan a cabo dichas asociaciones e interconexiones que formulan los nuevos enunciados. $Y$ es justo en ese instante donde radica la importancia del cuerpo. Sin un cuerpo presente, sin unos brazos, unas manos, y unos ojos, esas conexiones y vinculaciones serían inalcanzables en su totalidad, pues no se podría realizar el acto de seleccionar los documentos. Y no solo los teóricos se olvidan de la importancia de la presencia del cuerpo, sino que incluso los mismos comisarios se olvidan de ello, pues el mismo Aimar Arriola subrayaba en una entrevista la importancia de la mirada y del acto de visibilizar los documentos correspondientes del archivo para la producción de nuevos significados en las salas expositivas obviando la presencia y relevancia de los cuerpos de los visitantes en el mismo proceso (2016). Así, a causa de la sobreestimulación del diseño dispositivo, el ojo y la mirada quedaban saturadas ante la cantidad de información expuesta en la sala, y sin la ayuda del cuerpo y de las manos para la selección de los documentos, la activación y la creación de enunciados era prácticamente inexistente, pues los cuerpos solamente podían pasear en el espacio.

Otra de las claves para entender la importancia del cuerpo a la hora de activar el archivo nos la facilita Rolnik. A pesar de que la autora analiza el archivo desde el punto de vista de la creación, afirma rotundamente que los requisitos indispensables para activar el contenido de los mismos son las acciones de pensar y de crear. Dos acciones que solamente se pueden realizar a través de una actividad sensorial y por lo tanto, no solo mediante la perceptiva. De esta forma, lo que Rolnik está subrayando es que el pensamiento y la creación son dos acciones que se realizan a partir de nuestro cuerpo -en su totalidad-, sus efectos y afectos, y no solamente a través de la mirada, pues es así como se integran en nosotros, a pesar de que desde la creación de la modernidad occidental las ideas impuestas haya sido otras ${ }^{16}$. Los museos, como instituciones propagadoras de estas últimas ideas, no hicieron sino aplicarlas en sus salas, unas reglas que han llegado hasta nuestros días. Así en ellas, los espacios de actuación para el cuerpo y los sentidos son prácticamente nulos y el único sentido que tiene autorización para actuar es la mirada, que alimenta más, el campo del deseo, -vinculado a la idea de posesión- que la del conocimiento ${ }^{17}$ con el consiguiente control y poder que acarrea.

De esta forma, por una parte es evidente la necesidad de introducir el cuerpo y su materialidad en el espacio 
expositivo para poder activar en su totalidad la capacidad teórico-crítica del archivo, que es la única forma de que el archivo sea efectivo en la creación de experiencias crítico-poéticas, evitando su monumentalización y su objetualización ${ }^{18}$, ya que solo de esta forma será posible crear otras historias y evitar la creación de nuevos paradigmas totalitarios y hegemónicos, con la intención de desobstruir el futuro, y poder curar los posibles traumas que tenemos inscritos en la memoria de nuestros cuerpos. Pero por otra parte, también queda en evidencia la dificultad de crear espacios expositivos apropiados donde la presencia del cuerpo en su totalidad es bienvenida por el gran peso de la tradición que todavía tienen las formas de exponer y por ello, reivindicamos la necesidad de huir de dichos modos de hacer, y de crear nuevas teorías que sustenten y apoyen estas nuevas prácticas. Así, la intención de este artículo es proponer una nueva teoría basada en la teoría matricial de Ettinger, que funcione como punto de partida para una nueva aproximación hacia las instalaciones expositivas que pretendan activar los archivos, posibilitando la imbricación del uso del mismo con la presencia del cuerpo.

\section{El archivo desde lo matricial}

La teoría matricial de Bracha L. Ettinger intenta exponer y describir el sujeto desde lo simbólico femenino-maternal-matricial como parte de una red que sostenemos y nos sostiene, mediante la cual presenta la complejidad de las relaciones y vinculaciones humanas. En este punto, debemos mencionar que aunque Ettinger desarrolla esta teoría en relación a las conexiones humanas, en este apartado se pretende imbricarla en otro campo de estudio ${ }^{19}$, el del análisis de exposiciones, y utilizarla como medio para la comprensión de la vinculación que se da entre el cuerpo y el archivo. $Y$ es que la autora habla de recuperar un lugar de origen a través de su teoría (Mendizabal, 2016: 27) y, tal como mencionamos, si el archivo es algo, es precisamente un lugar de origen.

El punto de partida de ese lugar de origen, según Ettinger, sería la acción de haber nacido, por la cual en nuestro cuerpo-psique quedaría permanentemente trazado un encuentro compartido que no se pierde a lo largo de la vida y que continúa conectándonos. En ese primer encuentro co-habitan y se trans-conectan un Yo (pre-sujeto, el que es creado), y un no-Yo (trans-sujeto, a partir del cual es creado), para así después co-emerger de forma recíproca y asimétrica (Mendizabal, 2016: 27).

Ese primer momento del que habla Ettinger lo podemos asociar con el momento de la escritura, de la creación de los documentos que conformarán a posteriori el archivo. La escritura, el documento, sería el Yo, el ente creado, y el escritor-cuerpo, el no-Yo a partir del cual el documento sería creado, pues de ese primer momento del acto de la escritura derivan todos los documentos que componen un archivo. A partir de ese primer momento del encuentro, de su creación, el documento (Yo) y el escritor (no-Yo), co-habitan, se trans-conectan, y de alguna forma se co-emergen de forma recíproca, ya que la misma escritura y el documento final van dándole forma a las ideas trazadas del escritor; y asimétrica, pues en el acto de la escritura y conformación de documentos siempre habrá un no-Yo-escritor que domina en cierto grado al Yo-documento.

Es más, Ettinger afirma que «en el espacio tiempo duración trans-subjetivo matricial tiene lugar una continuidad psíquica entre el sujeto y el no-Yo»20. En este caso, en relación a la escritura, esa continuidad psíquica estaría compuesta por las ideas, pero no de ideas superficiales, sino de las ideas que han sido arraigadas totalmente en el cuerpo, en los cuerpos, y que por ello son compartidas por todos los sujetos de una misma sociedad, de un mismo contexto. Así, un documento-yo está totalmente vinculado a su particular no-yo-escritor, pero de la misma forma podrá asociarse a otros documentos-yos, pues comparten ese primer momento de creación, y a otros no-yos, por estar vinculados a través de la continuidad psíquica que menciona Ettinger. Es más, incluso el mismo proceso de co-emergencia, tras el cual se van creando y trazando las diferentes ideas y enunciados que después conformarán el texto, no se terminará en el mismo texto o documento, sino que el proceso de esa co-emergencia de enunciados seguirá desplegándose. Para ello, según Ettinger, se requiere de encuentros-acontecimientos trans-subjetivos, es decir, encuentros-acontecimientos donde los no-Yo (escritor-es, cuerpos) adquieran el protagonismo, y se relacionen con otros no-Yoes-cuerpos-escritores, pero también con otros Yoes-documentos (que conforman el archivo). A partir de estos encuentros-acontecimientos se van remodelando y articulando así 
los diferentes núcleos y nódulos de una red (que se darán en los cuerpos) que, según Ettinger, llevan las diferentes trazas de otros encuentros-acontecimientos anteriores. Es decir, tal y como lo resume Mendizabal «lo que nos ocurre en un encuentro se va transmitiendo y transfiriendo y transformando a lo largo de otros encuentros matriciales» (2016: 27-28).

En este punto, la concepción del archivo se entroncaría con las teorías foucaultianas, ya que según hemos visto, al estar conformado por encuentros-acontecimientos infinitos y en continua co-emergencia, el espacio matricial no es un espacio-contenedor, ni un espacio que se pueda acotar, sino que sería un ente inabarcable que posibilitaría solo aproximaciones parciales y fragmentadas. Sin embargo, Ettinger va más allá y afirma que los mencionados encuentros matriciales no se llevan a cabo entre todos los posibles Yoes (documentos), y no-Yoes (cuerpos), y que esto no sucede solamente por la inabarcabilidad, sino que depende de otro factor: los afectos. Así, estos encuentros-acontecimientos solamente se dan cuando los nódulos de las redes resuenan, es decir, cuando se crea el enunciado, que será cuando estos afectos se activan; es decir, cuando se produzca compasión, asombro y/o respeto, entre los cuerpos (no-Yo) y documentos (Yo) (Mendizabal, 2016: 28-41).

De esta forma, Ettinger nos ofrece una herramienta muy eficaz desde la que comprender no solo la vinculación existente entre los documentos que componen el archivo y los cuerpos, sino también su importancia a la hora de activar el archivo y crear nuevos, diferentes e ilimitados enunciados. Nos facilita incluir en el análisis la manera en que los cuerpos deben aproximarse al archivo y documentos para que los nodos y vínculos que se creen sean efectivos; una teoría donde tienen cabida tanto el cuerpo físico, como los sentimientos y situaciones particulares de cada cuerpo-sujeto-espectador que condicionan completamente cada experiencia. A través del cuerpo, Ettinger abre incluso la oportunidad para que esos vínculos y conexiones sean una acción dependiente de la elección personal, ya que una vez siendo conscientes de nuestros propios afectos, recae sobre el no-yo-cuerpo-sujeto-espectador la decisión de abrirse a dichos afectos y de crear nodos y vínculos (Mendizabal, 2016: 35-45), es decir, de crear o no los enunciados.

$Y$ aunque el hecho de abrirse a dichos afectos se trate de una toma de decisión, no es solo una decisión intelectual, sino que los cuerpos tienen que adoptar una posición subjetiva de apertura, es decir, los cuerpos-sujetos-espectadores deben auto-fragilizarse. Este concepto significa tomar una posición que posibilite abrirse, desprotegerse; implica ser vulnerable, exponerse, dar un paso atrás hacia otra perspectiva. Esta auto-fragilización ha de ser recíproca, pues ninguna de las dos partes tiene que estar a la defensiva. Así, se creará un espacio amplio y profundo, donde a través de esa auto-fragilización habrá una verdadera oportunidad para conectar con lo otro, para poder entrar hacia el (los) yo(s)-documento(s) (Mendizabal, 2016: 32-35). Y así, verdaderamente el cuerpo y la mente tendrán la oportunidad de abrirse a lo nuevo, a lo otro, y a los otros (documentos), y podrán verdaderamente crear nuevos significados y enunciados, aunque siempre sin dejar la identidad atrás, lo que abre la puerta a las creaciones de nuevos significados y enunciados desde esa identidad primera del cuerpo-sujeto-espectador.

Para llevar a cabo esa auto-fragilización, Ettinger considera necesarios dos elementos matriciales. Por una parte, una necesaria confianza, que según la autora es un tipo de amor, de Eros; aunque por desgracia, actualmente confiar se ha convertido en algo casi sospechoso (Mendizabal, 2016: 29-35), difícil de lograr, sobre todo frente a lo desconocido. El otro elemento matricial sería el carriance (sostener en el tiempo), un sostener o sujetar que ya aparece implícitamente en el mismo concepto sujeto-cuerpo-persona. Soy sujeto, porque he sido sostenido/sujetado en el tiempo, y porque sujeto/sostengo a otros también. Así, se trata de otro acto recíproco y asimétrico. Somos seres sostenidos-cuidados que a su vez sostenemos-cuidamos. Y no solo sostenemos a otros no-Yoes-cuerpos, sino que también a los Yoes que, de nuevo trayéndolo al terreno archivístico, significaría la sujeción y el sostén de los documentos que conforman el archivo. Una sujeción y un sostener-cuidados tanto simbólicos como también físicos, que como bien hemos mencionado serían totalmente necesarios para auto-fragilizarnos, y así poder realizar los encuentros-acontecimientos, vínculos y conexiones y, por ende, crear nuevos enunciados; que a su vez nos sujetan, y nos constituyen.

Así, gracias a la teoría matricial, ha quedado claro que para activar el archivo y crear nuevos enunciados tienen que darse ciertos requisitos afectuales y efectuales entre los cuerpos y el archivo; y que para ello, la forma que adquiera su instalación será decisiva. 
Último paso: La materialización formal

A pesar de que el análisis del proyecto Anarchivo Sida nos haya podido servir para identificar los elementos y decisiones que condicionaron la percepción y uso del archivo en el espacio expositivo, no es mi intención reformalizar su instalación aplicando la teoría matricial, ya que la extensión del artículo no me lo permitiría. No obstante, expondré un ejemplo posible, focalizándome en el diseño expositivo, con la intención de mostrar la viabilidad de la aplicación formal de la teoría.

Si redujera la teoría matricial a tres conceptos para poder aplicarla en una instalación, serían las siguientes: confianza, autofragilización, y sujeción. Así, como veremos a continuación, el diseño expositivo del proyecto no facilitaba ninguno de los tres requisitos, y una de las decisiones que condicionó este desenlace fue el uso de la madera en su medida estándar de tablón para ello. El objetivo de esta decisión era mimetizar el interior de la sala expositiva con el resto del edificio de Tabakalera, ya que se trata de un material que se utiliza en este espacio. Pero dentro del espacio expositivo las piezas adquirían una presencial monumental, creando un espacio totalmente jerarquizado, que no generaba ni confianza ni comodidad, donde los cuerpos se sentían en constante alerta, ya que al encontrarse en un espacio representacional, su presencia como cuerpo estaba betado. En cambio, si el diseño expositivo hubiera sido realizado a la escala del cuerpo humano, seguramente su monumentalidad hubiese desaparecido, la percepción del espacio no hubiera sido tan representacional ni jerárquica sino más horizontal y real donde habría más posibilidad de que los cuerpos se sintiesen más cómodos, y los documentos hubieran adquirido más protagonismo que los muebles que los sujetan. Este simple hecho podría haber alentado a los visitantes a quedarse, a tomárselo con calma, y a moverse con más naturalidad.

Otro elemento a tener en cuenta sería la forma en la que los documentos se exponen sobre los muebles. Una vez conseguido un espacio cómodo para los cuerpos y los documentos, seguidamente es necesario crear una atmósfera apropiada para la posible autofragilización de ambos. En este caso, en la instalación de Anarchivo Sida no había ningún tipo de barrera física (vitrinas o cristales) que separaban los cuerpos y los documentos, pero en algunos casos parecía que varios documentos estaban pegados a las mesas mo- numentales, dando la sensación de que estaban ahí simplemente para ser mirados, lo cual tampoco invitaba a que los visitantes tocaran los documentos con sus propias manos. En cambio, se podrían autofragilizar los documentos, jugando con el orden y así exponiendo los documentos no de una manera ortogonal y pulcra, sino algo desordenados, dando la sensación de haber sido ya manipulados por otros visitantes. De esta forma se conseguiría autofragilizar los documentos expuestos, y al mismo tiempo incrementar la sensación de confianza en los visitantes, lo cual les podría ayudar a dar el paso de su propia autofragilización. Esta acción consistiría en trasladarse de su espacio de confort, en el que siguen las reglas preestablecidas de los espacios expositivos, a un espacio de riesgo, en el que transgrediesen las reglas tradicionales y empezasen a realizar actos, a priori prohibitivos, como podría ser tocar los documentos expuestos.

Así, y viendo que no hay ningún tipo de represalia por adquirir nuevos comportamientos dentro del espacio expositivo, se incremente la confianza, y los cuerpos-espectadores podrían empezar a coger, sostener y leer diferentes documentos hasta aparecer distintos tipos de afectos entre ellos y así crear nuevos enunciados y activar el contenido del archivo.

\section{Conclusión}

Hemos analizado la instalación del proyecto Anarchivo Sida que buscaba cuestionar las estructuras de la producción artística, generando al mismo tiempo nuevos discursos históricos donde el cuerpo ocupara un espacio prioritario. Para llevar la propuesta al marco expositivo, los comisarios se han servido del formato archivístico siguiendo las teorías de Derrida, Pedro G. Romero y Foucault con la intención de crear nuevos discursos no hegemónicos, sino casi contra-archivísticos respecto a los archivos oficiales, para así poder cuestionar las construcciones históricas patriarcales.

Pero al observar el caso de estudio nos percatamos que para la activación de dichos archivos se necesita de un medio específico: del cuerpo-espectador. En el espacio expositivo, donde el ojo está sobreexpuestos a sobreestimulaciones para poder llevar a cabo la construcción de enunciados y por ende de conocimiento, el espectador necesita de la articulación total de su cuerpo para poder 
subvertir el régimen hegemónico de construcción de conocimiento. Así, aunque la instalación del dispositivo se centrara en los movimientos de dichos cuerpos dentro del espacio expositivo para con los soportes de los materiales, y lograra de alguna manera los diferentes tipos de interacciones previstos, los soportes adquirían demasiada entidad en comparación a los materiales y documentos expuestos, ya que el archivo no había sido pensado para articularse a su vez con esos mismos cuerpos. La razón de este suceso fue que las teorías en las que los comisarios se habían centrado a la hora de formalizar y definir el archivo excluían completamente la figura del cuerpo en sus discursos. Vemos por lo tanto que en la lógica comisarial no solo la instalación es fundamental para conseguir que el archivo funcione de una manera crítica, sino que para que la instalación funcione, las teorías en las que se basan los discursos comisariales tienen que ser también las adecuadas a su propósito.

En contraste, la teoría matricial de Ettinger no solo incluye la importancia del cuerpo en relación al archivo para crear sus múltiples enunciados, sino que al mismo tiempo proporciona propuestas que se pueden aplicar en el ámbito comisarial, específicamente en la instalación de los archivos, para que estos lleguen a ser efectivos en relación a los cuerpos-espectadores, y así mantener activo su potencial de crear enunciados. Pues por una parte, según hemos analizado, se necesita tanto la auto-fragilización o vulnerabilidad de los no-Yo-cuerpos-espectadores, como también de los documentos. Y esta vulnerabilidad o auto-fragilización de los documentos solamente se puede lograr a través de su instalación, es decir, creando un espacio donde gracias a la confianza-cuidado recíproca, el no-yo-cuerpo-espectador pueda acceder directamente a dichos documentos mediante todo su cuerpo, sin ningún tipo de intermediación entre los dos, sin ningún tipo de plástico, pegamento ni vitrina, para que el cuerpo pueda sostener los documentos del archivo, tanto simbólicamente como físicamente. Es así como, según Ettinger, se creará un espacio donde se podrán realizar los encuentros-acontecimientos entre el Yo-documento y el no-yo-cuerpo-espectador, y mediante los cuales se crearán a su vez los diferentes enunciados.

Durante las últimas décadas se han realizado múltiples proyectos comisariales que, apoyados en las teorías feministas, han utilizado el archivo como método expositivo con la intención de cuestionar los discursos totalitarios y oficiales que la historia patriarcal ha dado por verdaderos durante tantos años. Estos proyectos han tenido que enfrentarse a diferentes problemáticas, entre ellas algunas específicas en relación al archivo, a su conceptualización y su tratamiento formal dentro del espacio, ya que no han sido muchas las tentativas de reenfocarlo desde una perspectiva feminista que a la vez posibilitaran repensar su uso expositivo de una manera eficaz. Este estudio, basándose en la teoría matricial de Ettinger, trata de abrir una vía hacia la aproximación, conceptualización y formulación del archivo desde las teorías feministas y desde el cuerpo, para que a su vez pueda servir como herramienta y método expositivo eficaz para cuestionar los discursos hegemónicos, y posibilitar sistemas alternativos para la construcción de enunciados, historias y conocimiento.

\section{Notas}

1 A partir de ahora nos referiremos a Tabakalera-Centro Internacional de Cultura Contemporánea, como Tabakalera

2 Equipo Re es una plataforma de investigación interdisciplinar formada por Aimar Arriola, Nancy Garín y Linda Valdés, que están interesados principalmente en articular los espacios artísticos y las acciones críticas, para el cual divagan entre el archivo, encuentros, talleres, exposiciones, construcciones de cartografías y producción de narrativas (<https://equipo-re.org/>).

3 Según Laura Malosetti Costa, en la introducción a la traducción en castellano de Visión y diferencia. Feminismo, feminidad e historias del arte de Griselda Pollock: "El canon de la historia del arte es uno de los más virulentos y "virilentos"» (Pollock, 2015: 15) pues la historia del arte siguió aceptando dicho canon incluso ya cuando en otras disciplinas se estaba cuestionándolo».

4 Dentro de estas tentativas de cuestionar el canon, indudablemente cabe mencionar el gran trabajo realizado por Griselda Pollock.

5 La relación entre las prácticas artísticas y el archivo ha sido extensamente analizada por Anna Maria Guasch en su libro Arte y Archivo, 1920-2010. Genealogías, tipologías y discontinuidades (2011).

6 Según Morandeira la violencia que ejerce el archivo es triple: «la violencia extractiva, que expropia documentos y memorias; la violencia epistemológica, que ordena el archivo (y el mundo); y la violencia del Estado, que retiene los documentos fuera del alcance de los ciudadanos" (2016: 3).

7 Una de las autoras más relevantes en cuanto a este campo de estudio ha sido Griselda Pollock. Entre sus publicaciones se podrían destacar Visión y diferencia. Feminismo, feminidad e historias del arte y Differencing the Canon. Feminist Desire and the Writing of Art's Histories, entre otros. 
8 Se trata de una apropiación del término ya utilizado por Derrida en su ensayo Mal de archivo, mediante el cual Pollock vincula el concepto del archivo a la construcción selectiva y exclusiva de la historia del arte, redefiniéndolo así también como núcleo desde donde se articulan las normas de representación, deseos y fantasías (Osorez, 2017: 125-126)

9 ROLNIK, Suely, «Furor de Archivo» [conferencia]. En: <http://hidvl.nyu.edu/video/003617137.html> (fecha de consulta: 16-7-2019).

10 OSBORNE, Thomas (1999), «The Ordinariness of the Archive», History of the Human Sciences n. ${ }^{\circ}$ 12, p. 53, mencionado en Danbolt, 2014 : 103.

11 EQUIPO RE., "Anarchivo Sida». En: <https://equipo-re.org/proyectos-en-curso/proyecto-sida/> (fecha de consulta: 22-2-2018).

$12<$ www.anarchivosida.org> (fecha de consulta: 22-2-2018).

13 NOGUEIRA, Carme, «Anarchivo Sida. Dispositivo de sala». En: <http://www.cntxt.org/node/139> (fecha de consulta: 23-2-2018).

14 lbíd.

15 EQUIPO RE., «Anarchivo Sida» [conferencia]. En: <https://vimeo.com/62595679> (fecha de consulta: 24-2-2018).

16 ROLNIK, Suely, «Furor de Archivo» [conferencia]. En: <http://hidvl.nyu.edu/video/003617137.html> (fecha de consulta: 16-7-2019).

17 En relación a esto, Simon Sheik propone el concepto de economía del deseo. Gracias al cual el museo logra envolver al espectador para quedarse atrapado al mismo tiempo, entre las relaciones de poder y conocimiento. Es por esta razón, según Sheik, por lo que el museo consigue emitir así cómodamente, sus discursos unilaterales y totalitarios (Sheik, 2007)

18 ROLNIK, Suely, «Archivomanía». En: <http://www.aica-paraguay.com/?p=2240\&paged=3> (fecha de consulta: 16-7-2019).

19 Fue la misma Ettinger quien en una conversación llevada a cabo en la Universidad de Leeds en 2015 como actividad previa a la bienal de Estambul, proponía públicamente que lleváramos la teoría matricial a cualquier campo, a todos los campos, o a aquel campo donde sentíamos que nos conectaba y podíamos llevarla (Ettinger, 2015). Istanbul in/+ Leeds Event 4: Reading with Bracha Ettinger [Conferencia]. En: <https://www.youtube.com/watch?v=gmekeulqdn0> (fecha de consulta: 8-2-2016). Mencionado en Mendizabal, 2016.

20 Ettinger, «(M)Other Re-spect: Maternal Subjectivity, the Ready-made mother-monster and The Ethics of Respecting» en Studies in the Maternal 2 (1) (2010: 12), mencionado en Mendizabal, 2016: 27.

\section{Bibliografía}

ANSA, Garazi (2017), «Análisis del archivo como método para la elaboración y transmisión de nuevos discursos históricos a través del caso de estudio", Re.Act.Feminism \#2 - A Performing Archive, AusArt Journal for Research in Art, vol. 5, n. ${ }^{\circ}$ 1, pp. 71-90.

ARRIOLA, Aimar (2016), «Por fuera. Un ensayo de relaciones desde el Anarchivo sida» [conferencia]. En: <https://www.youtube.com/watch?v=N2EK4NUr9zo> (fecha de consulta: 28-2-2018).

ARRIOLA, Aimar, GARíN, Nancy y VALDÉS, Linda (2016), «Anarchivo Sida», en URIARTE, Jone A. (coord.), Orriak 3, Tabakalera, Donostia, pp. 20-27.

BENNET, Tony (1998), The Birth of the Museum. History, Theory, Politics, Routledge, Nueva York.

DANBOLT, Mathias (2014), «Arresting performances", en KNAUP, Berrina and STAMMER, Beatrice E. (eds), Re.Act.Feminism. A performing archive, Verlang für Moderne Kunst y Live art development agency, Nüremberg y Londres, pp. 96-108.

DERRIDA, Jacques (1997) Mal de archivo. Una impresión freudiana, Editorial Trotta, Madrid.

DRESSLER, Iris, «Entreacto». En: <http://www.editorialconcreta.org/-ENTREACTO-148-> (fecha de consulta: 26-2-2018)

EQUIPO RE., «Anarchivo Sida». En: <https://equipo-re.org/proyectos-en-curso/proyecto-sida/> (fecha de consulta: 22/2/2018).

EQUIPO RE., «Anarchivo Sida» [conferencia]. En: <https://vimeo.com/62595679> (fecha de consulta: 24-2-2018).

FOUCAULT, Michel (2002), La arqueología del saber, Siglo Veintiuno editores, Buenos Aires.

LERNER, Gerda (2018), La creación del patriarcado, Katakrak Liburuak, Pamplona.

MENDIZABAL, I. (2016), Estructura y materialidad del acontecimiento pedagógico -Un primer contacto con/desde la teoría matricial de Bracha L. Ettinger (TFM). EHU/UPV, Bilbao.

MORANDEIRA, Julia (2016), «Fiebre del archivo», en URIARTE, Jone A. (coord.), Orriak 3, Tabakalera, Donostia, pp. 3-6.

MULVEY, Laura (2001), «Placer visual y cine narrativo», en WALLIS, Brian (ed.), Arte después de la modernidad. Nuevos planteamientos en torno a la representación, ediciones Akal, Madrid, pp. 365-377.

NOGUEIRA, Carme, «Anarchivo Sida. Dispositivo de sala». En: <http://www.cntxt.org/node/139> (fecha de consulta: 23-2-2018).

OSOREZ, Leticia (2017), «Cuerpo género y arte contemporáneo tucumano: una propuesta teórica para el análisis de obras», Revista científica de la Redcom, vol. 2, n. ${ }^{\circ}$ 4, pp. 123-130.

POLLOCK, Griselda (1999), Differencing the Canon. Feminist Desire and the Writing of Art's Histories, Routledge, New York. 
POLLOCK, Griselda (2008), «Desde las intervenciones feministas hasta los efectos feministas en las historias del arte. Análisis de la virtualidad feminista y de las transformaciones estéticas del trauma», en ARAKISTAIN, Xavier y MÉNDEZ, Lourdes (eds.), Producción artística y teoría feminista del arte: nuevos debates I, CC Montehermoso, Vitoria, pp. 42-63

- (2015), Visión y diferencia. Feminismo, feminidad e historias del arte, Fiordo No Ficción 01, Buenos Aires

ROLNIK, Suely (2009), «Furor de Archivo» [conferencia]. En: <http://hidvl.nyu.edu/video/003617137.html> (fecha de consulta: 16-7-2019).

- (2011), «Archivomanía». En: < http://www.aica-paraguay.com/?p=2240\&paged=3> (fecha de consulta: 16-7-2019).

SALOMON, Nanette (1998), «The Art historical Canon», en PREZIOSI, Donald (ed.), The art of art History: A critical antology, Oxford University Press, Oxford, pp. 344-355.

SPIVAK, Gayatri C. (2006), «The Rani of Sirmur: An Essay in Reading the Archives (Introduction)», en MEREWETHER, Charles (ed.), The Archive (Documents of Contemporary Art), Whitechapel Gallery y The MIT Press, Londres y Cambridge, pp. 163-169.

Whitechapel Gallery y The MIT Press, Londres y Cambridge, pp. 163-169. 